\title{
Optical second-harmonic probe for ultra-high frequency on-chip interconnects with benzocyclobutene
}

\author{
M. Nagel, ${ }^{\text {a) }}$ C. Meyer, H.-M. Heiliger, T. Dekorsy, and H. Kurz \\ Institut für Halbleitertechnik II, Rheinisch-Westfälische Technische Hochschule (RWTH) Aachen, \\ D-52056 Aachen, Germany \\ R. Hey and K. Ploog \\ Paul-Drude-Institut, D-10117 Berlin, Germany
}

\begin{abstract}
We report the application of electric field-induced second-harmonic (EFISH) generation for time-resolved measurement of ultra-fast electrical pulses propagating on thin-film microstrip lines fabricated with a polymer based on benzocyclobutene as a dielectric layer on a silicon substrate. This contactless field detection enables the characterization of electric pulses on the transmission line up to $1.95 \mathrm{THz}$. In comparison to external electro-optic sampling the EFISH technique provides a better characterization tool concerning time-resolution and noninvasiveness.
\end{abstract}

Interconnects become more and more limiting components in circuit performance as operating speeds of single transistors improve and structure dimensions shrink. ${ }^{1}$ Strong improvements in the propagation of high frequency signals have been made by inserting polymer materials as dielectric layers. ${ }^{2-4}$ Polymers have been synthesized to combine several properties of central importance. They have to be compatible with thin-film fabrication technology. They should have a low dielectric constant and good adhesion to several metals and substrate materials. In addition, low dielectric loss and a high degree of planarization with a wide range of layer thickness are required. With these characteristics combined in one material they provide a superior potential compared to conventional dielectrics like silicon dioxide, silicon nitride, or high ohmic silicon. Here we demonstrate an important advantage of these polymers, i.e., that the polymer itself can be utilized as a probe for contactless and noninvasive on-chip measurements of high frequency electric fields up to $1.95 \mathrm{THz}$ via electric-field-induced second harmonic (EFISH) generation.

In past years the most commonly used millimeter-wave interconnects were based on coplanar waveguides (CPWs) on $\mathrm{Si}$ or GaAs substrates. ${ }^{5}$ These transmission lines suffer from relatively high attenuation due to metallic losses at small dimensions. Conventional CPWs possess optimized propagation properties if high resistivity substrates are used. ${ }^{6}$ For frequencies below $30 \mathrm{GHz}$ and at small-scale integration levels, a conventional microstrip line (MSL) with ground metallization on the backside of the substrate is often employed. Because the signal-conductor width has to be matched to the substrate thickness, dimensions of the MSL are large compared to CPWs. For the same reason modal dispersion increases, making MSLs unsuitable for the (sub)$\mathrm{mm}$-wave range. Another type of waveguide is the thin-film microstrip line (TFMSL) with the signal and ground conductor located on top of the wafer surface separated by a thin

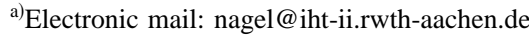

dielectric layer. By using polymeric layers, the organic material can be optimized to several requirements by manipulating its molecular structure. We examine TFMSLs of Cyclotene, a polymer based on benzocyclobutene (BCB) with attractive material properties for interlevel dielectrics in high-speed interconnect systems and multichip modules (MCMs). ${ }^{7-9}$ It has a low relative dielectric constant of 2.7 and exhibits good planarization characteristics. Single layer coatings can be made by the spin-on technique with a thickness from 1 to $25 \mu \mathrm{m}$ on a Si based circuit. The TFMSLs exhibit exceptionally low modal dispersion up to frequencies of $1.0 \mathrm{THz}$, as observed in studies based on electro-optic sampling. ${ }^{4}$ Since no field components enter the $\mathrm{Si}$ substrate in the case of the TFMSL, the effective permittivity is low $\left(\leqslant 2.2\right.$ at $100 \mathrm{GHz}$, for CPWs on silicon $\varepsilon_{r, \text { eff }}$ is about 3 times higher). Comparative studies of TFMSLs with silicon dioxide and TFMSLs with BCB as a dielectric layer demonstrate the following main advantages given by the polymer: (i) easy control of layer thickness within a favorable range leading to reduced attenuation, and (ii) lower dispersion due to the higher relative dielectric constant of $\mathrm{SiO}_{2}\left(\varepsilon_{r}=3.9\right) .{ }^{10}$ Compared to CPWs a standard $50 \Omega$ impedance line can be achieved for TFMSLs with smaller lateral dimensions of the signal conductor, allowing a more compact circuit design. ${ }^{11}$

High-speed millimeter-wave integrated circuits (MMICs) with operation frequencies up to $300 \mathrm{GHz}$ require measurement techniques with high temporal and spatial resolution. All electronic equipment like network analyzers or sampling oscilloscopes are limited to a $110 \mathrm{GHz}$ practical bandwidth. Optical time-domain measurement systems, however, can reach the highest temporal resolutions up to $150 \mathrm{fs}$ and spatial resolutions of $\sim 1 \mu \mathrm{m} .{ }^{12}$ An early optical approach for measuring voltage waveforms in an integrated circuit relied on sensing the induced electric field in an external electro-optic (EO) crystal brought near the circuit. ${ }^{13}$ However, this method introduces unwanted parasitics into the circuit $^{14}$ and is affected by the frequency response of the EO material itself. Later work which was done in this field directly used the birefringence of GaAs substrates for inter- 


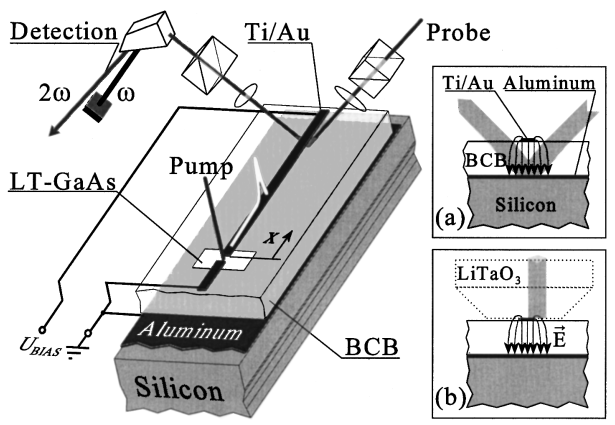

FIG. 1. Experimental EFISH setup for time-domain impulsive measurement on the TFMSL with an internal photoconductive switch. Insets: The schematic geometry in (a) internal EFISH generation and (b) external EO sampling probing.

nal EO sampling. ${ }^{15}$ However, the most important material for electric circuits-silicon-does not exhibit birefringence, so that internal EO sampling is not possible. Ti:sapphire lasers generating $100 \mathrm{fs}$ pulses of about $10 \mathrm{~nJ}$ pulse energy, however, were enabled to measure internal electric fields in crystalline silicon via EFISH generation. ${ }^{16-19}$ This technique does not require an external probe. It suffers, however, from a residual invasiveness as long as the laser energy is larger than the Si band gap. In this letter we demonstrate the first measurement of transient internal electric fields by EFISH generation in high frequency TFMSLs with BCB as a dielectric layer and nonlinear optical field probe. Hence, the method requires no additional probe and the invasiveness is significantly reduced in the weakly absorbing polymer.

The TFMSL, as drawn in Fig. 1, is fabricated on a lowresistivity (5-8 $\Omega \mathrm{cm}$ ) Si substrate. First, a 700-nm-thick Al ground metallization is deposited by electron beam evaporation. Then the polymer, commercially available Cyclotene (Dow Chemical 3022-63), is spin deposited to a thickness of $12 \mu \mathrm{m}$. Subsequently, the film is cured for $60 \mathrm{~min}$ at $250{ }^{\circ} \mathrm{C}$ in a $\mathrm{N}_{2}$ ambient. A 500-nm-thick film of low temperature grown (LT) GaAs with an $\sim 250 \mu \mathrm{m}^{2}$ area, is attached on the insulator via van der Waals bonding. The LT-GaAs is prepared via epitaxial liftoff and acts as a photoconductive (PC) switch. ${ }^{20}$ Finally a $3-\mu \mathrm{m}$-wide Ti/Au signal conductor of 700 $\mathrm{nm}$ thickness is placed on the top of the LT-GaAs. This Ti/Au signal conductor exhibits a $7-\mu \mathrm{m}$-wide gap for optical excitation.

For time-domain EFISH measurements we use a modelocked Ti:sapphire laser emitting $100 \mathrm{fs}$ optical pulses with a repetition rate of $75.6 \mathrm{MHz}$. For comparison EO sampling measurements are performed with a second Ti:sapphire laser with a $150 \mathrm{fs}$ optical pulsewidth. The operating wavelength is 805 and $750 \mathrm{~nm}$ for EFISH generation and EO sampling, respectively. Both setups are based on a pump probe scheme, where one pump pulse (10 $\mathrm{mW}$ average power) excites the 20-V-biased PC gap in the TFMSL and a temporally delayed probe pulse is used for the detection of the propagating electric field (Fig. 1). In the EFISH setup the probe pulses (45 $\mathrm{mW}$ average power) sample the electric transients within the polymer after a certain propagation distance. The laser beam is focused on the device to a spot diameter of $10 \mu \mathrm{m}$. The second-harmonic signal is detected by a gated single photon counting system. With EO sampling a $\mathrm{LiTaO}_{3}$ crystal is applied in contact to the device as a transducer. ${ }^{13} \mathrm{EFISH}$ gen-

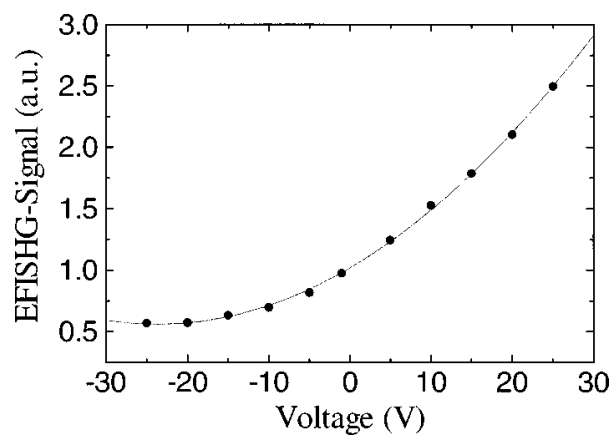

FIG. 2. Dependence of $I_{p, p}^{(2 \omega)}$ on the applied dc bias voltage between signal and ground conductor of the TFMSL. The solid curve is a parabolic fit to the data.

eration probes internal fields rather than the field components penetrating into air as in the case of EO sampling. These internal dielectric fields are by far more relevant for the characterization of the TFMSL.

In the SHG experiments we detect the $p$-polarized second-harmonic response $I_{p, p}^{(2 \omega)}$ generated by a $p$-polarized fundamental field $E_{p}^{(\omega)}$, where $p$ denotes the direction parallel to the plane of incidence. All time-resolved measurements are performed with the plane of incidence vertically to the signal conductor, as shown in Fig. 1, where a maximum response is obtained. The $s$-polarized SH response $I_{s, s}^{(2 \omega)}$ generated by an $s$-polarized fundamental field $E_{s}^{(\omega)}$ does not exhibit an EFISH signal. This polarization dependence is based on the fact that a third-order nonlinear polarization $P^{3}(\omega, \omega, 0)$ at the frequency $2 \omega$ is generated in the polymer, only when the quasi-dc field and the optical field have the same polarization. We calibrate the electric field-induced second-harmonic intensity in the bias voltage range of interest in a non-time resolved experiment. Figure 2 shows the relative EFISH signal $I_{p, p}^{(2 \omega)}(U) / I_{p, p}^{(2 \omega)}(0)$ versus the applied signal-to-ground conductor voltage, probed at a point for later time-domain measurement on the transmission line. The data show a parabolic dependence on the voltage with a minimum at $-23.5 \mathrm{~V}$. The shift from the origin is caused by the interference effect between the two SH contributions, which originates from symmetry perturbation at the polymer/ metal interfaces. This effect can be expressed as ${ }^{19} I^{2 \omega}(U)$ $=\left|A+B \cdot U e^{i \psi_{e l}}\right|^{2}$, where $A$ is the field-independent $\chi^{(2)}$ contribution, the second term the anisotropic field-dependent $\chi^{(3)}$ contribution, and $\psi_{e l}$ a phase difference between both. The fit parameters deduced from the data in Fig. 2 are $A$ $=1.00, B=0.029 \mathrm{~V}^{-1}$, and $\psi_{e l}=-74^{\circ}$. Because the fieldindependent contribution is relatively large and $\psi_{e l} \neq 90^{\circ}$ the SH signal is dominated by the mixed term in the fitting equation $\left[2 A B U \cos \left(-74^{\circ}\right)>B^{2} U^{2}\right]$ leading to a minimum of $I^{2 \omega}$ at $-23.5 \mathrm{~V}$. This behavior may be advantageous, because it yields a more linear sensitivity around the $0 \mathrm{~V}$ bias. We determine a noise amplitude of about $100 \mathrm{mV}$ at $0 \mathrm{~V}$ bias with a time constant $\tau=2 \mathrm{~s} \mathrm{(20} \mathrm{mV} \mathrm{with} \tau=100 \mathrm{~ms}$ for EO sampling). The sensitivity may be further increased with higher laser power, limited by the residual absorption and heating of the polymer.

Next, we demonstrate the application of the EFISH technique for time-domain measurement on TFMSLs with BCB as the dielectric layer in comparison to EO sampling. Time- 


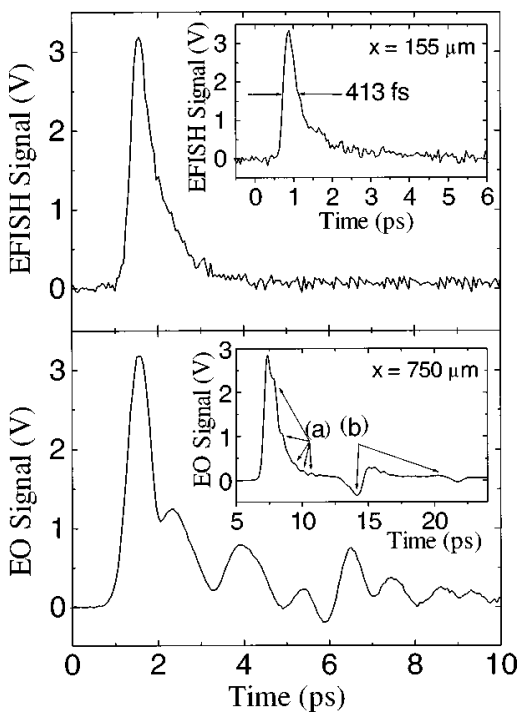

FIG. 3. Comparison of time-domain waveforms obtained using EFISH generation and EO sampling probed at $x=300 \mu \mathrm{m}$ distance from the PC switch (except for the insets). Marked dips in the EO sampling data point out (a) internal crystal reflections and (b) reflections between the crystal and the PC switch.

resolved electric field amplitudes from EFISH generation and EO sampling are shown in Fig. 3. The data are probed at a distance of $300 \mu \mathrm{m}$ from the PC gap. ${ }^{21}$ Because the EO voltage dependence is linear, the signal can be calibrated with a simple reference voltage measurement. The deviation from linearity of the EFISH sensitivity is less than $2.5 \%$ in the range of $\pm 5 \mathrm{~V}$ around the applied bias of $20 \mathrm{~V}$ at an amplitude of the signal of $\sim 3 \mathrm{~V}$. Thus the assumption of a linear dependence between the transient EFISH data and the voltage is justified. The time domain data include information on the attenuation and the dispersion of the TFMSL. ${ }^{4}$ Both signals are dominated by the short electrical pulse of 580 fs full width at half-maximum (FWHM) duration (EFISH, 665 fs for EO sampling). The most salient difference between EFISH generation and EO sampling is that the EFISH signal decays within $1 \mathrm{ps}$, while the EO signal is strongly modulated by several features, which last for more than 10 ps. Data from a second EO probe position at $x$ $=750 \mu \mathrm{m}$ are added to assign the origins of these contributions. The electric signals are reflected at the front edge of the $\mathrm{LiTaO}_{3}$ crystal and returned from the open end of TFMSL at the PC gap causing sequenced negative and positive dips on the backside of the signal. In addition the electric signals are reflected at each edge inside the crystal causing multiple positive peaks (see inset Fig. 3). Reflections from the unmatched end of the stripline do not appear within the shown time domain, because the line has a length of 11 $\mathrm{mm}$ at both sides of the switch. Although the EFISH signal exhibits a smaller signal-to-noise ratio than the EO signal, the noise from the EFISH data is by far less in amplitude than the artificial temporal structures in the EO signal. The inset in the EFISH data depicts the signal at $150 \mu \mathrm{m}$ distance from the PC switch. The pulsewidth detected at this point is only 413 fs FWHM, which corresponds to a bandwidth of $1.95 \mathrm{THz}$ at a $20-\mathrm{dB}$ drop in the frequency spectrum assuming a Gaussian pulse. The pulsewidth of $413 \mathrm{fs}$ is related to a free carrier lifetime of $250 \mathrm{fs}$ determined from the single-line pump-probe experiment. ${ }^{22}$ From EFISH measurements at various spatial delays a signal velocity of $\sim 0.2 \mathrm{~mm} / \mathrm{ps}$ is derived. This large value is mainly due to the low permittivity $\varepsilon_{r}=2.7$ of BCB.

In conclusion, we have demonstrated time-domain measurements on a polymer based TFMSL with EFISH generation. In comparison to external EO sampling this method has the essential advantage of noninvasiveness. The use of the polymer as a field probe opens the potential for the timeresolved characterization of arbitrary integrated silicon circuits.

The authors are grateful to G. Lüpke for discussion and Dow Chemical for supplying samples of Cyclotene. This work is supported by the DFG under Contract No. Ku 540/ 31-1.

${ }^{1}$ H.-M. Rein and M. Möller, IEEE J. Solid-State Circuits 31, 1076 (1996).

${ }^{2}$ S. Banba, T. Hasegawa, and H. Ogawa, IEEE Microwave Guid. Wave Lett. 1, 346 (1991).

${ }^{3}$ P. B. Chinoy and J. Tajadod, IEEE Trans. Compon., Hybrids, Manuf. Technol. 16, 714 (1993).

${ }^{4}$ H.-M. Heiliger, M. Nagel, H. G. Roskos, H. Kurz, F. Schneider, W. Heinrich, R. Hey, and K. Ploog, Appl. Phys. Lett. 70, 17 (1997).

${ }^{5}$ M. Y. Frankel, S. Gupta, J. A. Valdmanis, and G. A. Mourou, IEEE Trans. Microwave Theory Tech. MTT-39, 910 (1991).

${ }^{6}$ S. Alexandrou, R. Sobolewsky, and T. Y. Hsiang, IEEE J. Quantum Electron. 28, 2325 (1992).

${ }^{7}$ S. Bothra, M. Kellam, and P. Garrou, J. Electron. Mater. 23, 8 (1994).

${ }^{8}$ S.-F. Gong, J. Strandberg, H. Thiede, H. Hentzell, H. Hesselboom, and W. Karner, IEEE Trans. Compon., Hybrids, Manuf. Technol. 16, 735 (1996).

${ }^{9}$ G. A. Patrizi, M. L. Lovejoy, P. M. Enquist, R. P. Schneider, and H. Q. Hou, Thin Solid Films 291, 435 (1996).

${ }^{10}$ H.-M. Heiliger, M. Nagel, H. G. Roskos, H. Kurz, F. Schneider, and W. Heinrich, IEEE MTT-S Dig. 421 (1997).

${ }^{11}$ H.-M. Heiliger, M. Nagel, M. Setz, H. G. Roskos, H. Kurz, F. Schneider, and $\mathrm{W}$. Heinrich (unpublished).

${ }^{12}$ T. Pfeifer, H.-M. Heiliger, T. Löffler, C. Ohlhoff, C. Meyer, G. Lüpke, H. G. Roskos, and H. Kurz, IEEE J. Sel. Top. Quantum Electron. 2, 586 (1996).

${ }^{13}$ K. E. Meyer and G. A. Mourou, Series on Electrophysics (Springer, New York, 1985), Vol. 21, p. 46.

${ }^{14}$ W. Mertin, C. Roths, F. Taenzler, and E. Kubalek, IEEE MTT-S Dig. pp. 1351.

${ }^{15}$ K. J. Weingarten, M. J. W. Rodwell, and D. M. Bloom, IEEE J. Quantum Electron. QE-24, 198 (1988).

${ }^{16}$ O. A. Aktsipetrov, A. A. Fedyanin, V. N. Golovkina, and T. V. Murzina, Opt. Lett. 19, 1450 (1994).

${ }^{17}$ C. Ohlhoff, C. Meyer, G. Lüpke, T. Löffler, T. Pfeifer, H. G. Roskos, and H. Kurz, Appl. Phys. Lett. 68, 12 (1996).

${ }^{18}$ A. Nahata, J. A. Misewitch, and T. F. Heinz, Appl. Phys. Lett. 69, 746 (1996).

${ }^{19}$ C. Ohlhoff, G. Lüpke, C. Meyer, and H. Kurz, Phys. Rev. B 55, 7 (1997).

${ }^{20}$ E. Yablonovitch, D. M. Hwang, T. J. Gmitter, L. T. Florez, and J. P. Harbison, Appl. Phys. Lett. 56, 2419 (1990).

${ }^{21}$ The EO-crystal dimensions do not permit a closer approach, while the internal EFISH generation has no such restrictions.

${ }^{22}$ Care has to be taken concerning the determination of the carrier lifetime in LT-GaAs. See, G. Segschneider, T. Dekorsy, H. Kurz, R. Hey, and K. Ploog, Appl. Phys. Lett. 71, (1997). 